OPEN ACCESS

Edited by:

Badri Padhukasahasram,

Illumina, United States

Reviewed by:

David Vieites,

Consejo Superior de Investigaciones

Científicas (CSIC), Spain

Jinfeng Chen,

University of California, Riverside,

United States

Brian C. Verrelli,

Virginia Commonwealth University,

United States

*Correspondence:

Shi-Kang Shen

yunda123456@126.com

ssk168@ynu.edu.cn

Specialty section:

This article was submitted to Evolutionary and Population Genetics,

a section of the journal

Frontiers in Plant Science

Received: 02 June 2017 Accepted: 11 September 2017

Published: 26 September 2017

Citation:

Zhang $Y$, Zhang $X$, Wang $Y-H$ and Shen S-K (2017) De Novo Assembly of Transcriptome and Development of

Novel EST-SSR Markers in

Rhododendron rex Lévl. through

Illumina Sequencing.

Front. Plant Sci. 8:1664.

doi: 10.3389/fpls.2017.01664

\section{De Novo Assembly of Transcriptome and Development of Novel EST-SSR Markers in Rhododendron rex Lévl. through Illumina Sequencing}

\author{
Yue Zhang, Xue Zhang, Yue-Hua Wang and Shi-Kang Shen* \\ School of Life Sciences and Laboratory of Ecology and Evolutionary Biology, Yunnan University, Yunnan, China
}

Transcriptome sequences generated by next-generation sequencing (NGS) technologies can be utilized to rapidly detect and characterize a large number of gene-based microsatellites from different plants. Rhododendron rex Lévl. is a perennial woody species from the family Ericaceae and an endangered plant with high ornamental value endemic to Southwestern China. Nevertheless, the genetic and genomic information of $R$. rex remain unknown. In this study, we performed transcriptome sequencing for $R$. rex leaf samples, and generated large transcript sequences for functional characterization and development gene-associated SSR markers. A total of 164,242 unigenes were assembled and 115,089 (70.07\%) unigenes were successfully annotated in public databases. In addition, a total of 15,314 potential EST-SSRs were identified, and the frequency of SSRs in the R. rex unigenes was 9.32\%, with an average of one EST-SSR per $5.65 \mathrm{~kb}$. The most abundant type was repeated di-nucleotide (54.63\%), followed by mono- $(26.03 \%)$ and tri-nucleotide $(18.51 \%)$ repeats. Based on the SSR-containing sequence, 100 primer pairs were randomly selected and synthesized and used for assessment of the polymorphism. Thirty-six primer pairs were polymorphic and revealed polymorphism among 20 individuals from four $R$. rex populations. A total of 197 alleles were identified, with an average of 5.472 alleles per locus. The Polymorphism Information Content ranged from 0.154 to 0.870 , with a mean of 0.482 . The newly developed EST-SSR markers exhibited high transferability (58.33-83.33\%) among the six subgenera. Thus, these novel EST-SSR markers developed would provide valuable sequence resources for population structure, genetic diversity analysis, and genetic resource assessments of $R$. rex and its related species.

Keywords: Rhododendron rex, EST-SSRs, transcriptome sequencing, genomics, evolutionary adaptation

\section{INTRODUCTION}

Rhododendron L. is the largest woody plant genus, which contains at least 1,025 species and belongs to the Ericaceae family, and is widely distributed from the northern temperate zone, throughout tropical Southeast Asia, to northeastern Australia (Chamberlain et al., 1996). In China, more than 500 species of Rhododendron exist; of which, $70 \%$ are endemic and most species occur in the northwestern region of Yunnan provinces. Northwestern Yunnan has been recognized as a 
diversification and differentiation center of modern Rhododendron L. (Ma et al., 2013; Shen et al., 2015). Rhododendron species are not only the major components of alpine and subalpine vegetation but also recognized as famous woody ornamental plants worldwide (Yoichi et al., 2016). However, Rhododendron germplasm is at risk of extinction due to the high frequency of habitat disturbance and climate change (Wu et al., 2015). Thus, wild Rhododendron germplasm must be conserved to maintain the stability of ecosystems and promote sustainable resource utilization.

Next-generation sequencing (NGS), particularly de novo transcriptome sequencing, has become a cutting-edge method for high-throughput sequencing in life sciences research (Mardis, 2008; Davey et al., 2011; Zhou et al., 2016). NGS is an accurate and efficient technology with high throughput and low cost and thus can be used to discover comprehensive expressed sequence data for several non-model species. Transcriptome sequencing not only offers a simple and effective method of developing a large number of unigene-based SSR markers but also allows rapid and comprehensive analyses of the plant genome in many woody plants (Li et al., 2012; Tan et al., 2013; Chen L. Y. et al., 2015). NGS technologies have contributed to improvements in the fields of ecology, evolution, and conservation genetics by increasing the amount of large-scale genomic and transcriptomic data available for non-model woody plant species.

Many types of markers have been developed and used for genetic linkage, molecular-assisted breeding, genetic diversity analysis, and genetic quality detection (Paux et al., 2012; Wu et al., 2014). Simple sequence repeat (SSR) markers can be categorized into genomic SSRs, which are identified from random genomic sequences, and expressed sequence tag (EST) SSRs, which are identified from transcribed RNA sequences (Song et al., 2012). These tandem repeated sequences contain mono-, di-, tri-, tetra-, penta-, or hexa-nucleotide units, which possess abundant information, codominance, and loci specificity; as such, SSR markers are more convenient to use for detection compared with other molecular markers (Zhou et al., 2016). SSR makers have been widely used as a powerful tool in studies of population genetic diversity and genetic structure analysis (Yoichi et al., 2016). Inferring genetic variation patterns within a species is crucial to understand population structure, local adaptation, and diversity differences among populations (Marsden et al., 2012). Climatic/environmental factors and adaptive processes play an important role in the maintenance of lineage divergence of alpine and subalpine Rhododendron species (Cao et al., 2004; Hart, 2015). Thus, SSRs derived from expressed sequence tags (ESTSSRs) can be used in studies on local adaptation and effects of environmental variability because of the potential relationship of these tags to functional genes controlling a certain phenotype (Cordeiro et al., 2001; Varshney et al., 2005; Kumari et al., 2013). Moreover, EST-SSRs, which are developed from the transcribed regions in a genome, are more evolutionarily conserved and have higher levels of transferability to related species than non-coding genomic SSRs (Wei et al., 2011). However, few scholars have investigated the development of EST-SSRs through transcriptome sequencing analysis in Rhododendron, particularly for endemic species.
In this study, we used an Illumina sequencing platform for transcriptome sequencing analysis of RNA extracted from the leaves of $R$. rex Lévl., an endangered plant endemic to China (Fu and Jin, 1992). The sequencing data were assembled and annotated, and EST-SSR markers were also developed. Specifically, the aims of this study were to (1) characterize the transcriptome of $R$. rex; (2) determine the frequency and distribution of SSRs, develop EST-SSR markers and examine the level of polymorphism; (3) evaluate the transferability of the polymorphism EST-SSR markers to related species.

\section{MATERIALS AND METHODS \\ Plant Material and RNA Isolation}

Three R. rex plant materials were collected from the Baicaoling in Dayao County, Yunnan Province in October 2016 and sampled at least $15 \mathrm{~m}$ apart from each other. Fresh leaf samples were cleaned and immediately placed in liquid nitrogen until RNA extraction. For Illumina sequencing, total RNA was isolated from each sample by using TRIzol ${ }^{\circledR}$ Reagent (Invitrogen, CA, USA). RNA quality was verified using a Nanodrop 2000 (Thermo, USA). RNA Integrity Number value of the three samples were more than 7, as assessed using an Agilent 2100 Bioanalyzer (Agilent Technologies, CA, USA). The integrity of the qualified RNA was analyzed through agarose gel electrophoresis. More than $30 \mathrm{ng}$ of RNA was equally pooled from the three individuals for preparing a complementary DNA (cDNA) library.

\section{cDNA Library Construction and Sequencing}

The cDNA library for mRNA-seq was prepared using Illumina Truseq $^{\mathrm{TM}}$ RNA sample prep Kit (Illumina, CA, USA). The poly(A) tail structure of eukaryotic mRNA 3 ' end was isolated via magnetic oligo ( $\mathrm{dT}$ ) beads. mRNA samples were randomly sheared for transcriptome information analysis, added with fragmentation buffer and broken into $300 \mathrm{bp}$ fragments. The first-strand cDNA was formed via reverse transcription using reverse transcriptase and random hexamer primer using mRNA as a template. Then, second-strand cDNA was synthesized, forming a stable double-stranded structure. cDNA double-strand structure is cohesive end, and adding End Repair Mix results in a blunt ended structure. To select the proper templates for downstream enrichment, the products of the ligation reaction were purified on 2\% agarose gel (Certified Low Range Ultra Agarose). PCR amplification of 15 cycles was conducted to enrich the purified cDNA template using PCR primers PE 1.0 and 2.0 (Illumina) using DNA polymerase. The cDNA library was sequenced using Illumina HiSeq4000 sequencing platform at the Major Company (Shanghai, China). The images were automatically collected by base calling generation into FASTQ files (.fq).

\section{Sequence Assembly and Annotation}

By removing the adapter contaminants, reading too much poly$\mathrm{N}$, reading $<20$ bp to filter the raw sequencing data, screening out high-quality clean read data for de novo assembly, empty reads and reads with $Q<20$, using the program SeqPrep 
(https://github.com/jstjohn/SeqPrep). The GC content, sequence replication levels, and Q20 and Q30 values of the obtained clean data were calculated (Grabherr et al., 2011). In addition, the resulting cleaning data is de novo assembled by the short reading assembly program Trinity (http://trinityrnaseq.sourceforge.net/). This soft combines reads that have overlapping nucleic acid sequences to form contigs. Then, obtained contigs are assembled and sequences that cannot be extended at both end are defined as unigenes. The assembled unigenes were aligned using BLASTX with the public database $(E<1 \mathrm{e}-5)$, such as NCBI Nr, String, Swissprot, Kyoto Encyclopedia of Genes, and Genomes (KEGG), and Pfam, to obtain protein function annotation and classification information (Camacho et al., 2009). The gene ontology (GO) annotation information of these unigenes were obtained from the NCBI $\mathrm{Nr}$ database by using the program Blast2GO and contains molecular functions, biological processes, and cellular components (Conesa et al., 2005). Furthermore, these unigenes were categorized and analyzed in the COG database (http://www.ncbi.nlm.nih.gov/COG/) based on the COG information obtained from the string. The program WEGO (Ye et al., 2006) classified all unigenes based on the GO annotation information.

\section{Detection of the New EST-SSR Markers and Primer Design}

Potential SSRs were detected using the program MISA version 1.0 (http://pgrc.ipk-gatersleben.de/misa/). The mono-, di-, tri, tetra-, penta-, and hexa-nucleotides were designed with minimum repeat numbers of $10,6,5,5,5$, and 5 for the SSRs, respectively. With default parameter values, EST-SSR primers were designed using the Primer 3 (http://probes.pw.usda.gov/ cgi-bin/batchprimer3/batchprimer3.cgi). The designed EST-SSR primers were synthesized by Sangon laboratory (Shanghai, China).

\section{EST-SSR Amplification and Validation}

A total of 20 individuals from four populations (Supplementary Table S1) of $R$. rex were selected for polymorphism analyses using the obtained EST-SSRs. Whole genomic DNA of each individual was extracted from the dried leaves using the modified CTAB method (Doyle, 1991). PCR reactions were referenced and modified into a total reaction volume of $20 \mu \mathrm{L}$ containing $20 \mathrm{ng}$ of DNA, $2 \mu \mathrm{L}$ of $10 \times$ PCR buffer, $1.6 \mu \mathrm{L}$ of dNTPs $(10 \mathrm{mM})$, $0.4 \mu \mathrm{L}$ of each primer, $0.3 \mu \mathrm{L}$ of Taq DNA polymerase $(5 \mathrm{U} / \mu \mathrm{L}$; Takara, Shiga, Japan), and $14.3 \mu \mathrm{L}$ of double-distilled water. For each reaction, the following conditions were used: initial $4 \mathrm{~min}$ of denaturation at $94^{\circ} \mathrm{C}$, followed by 35 cycles of $40 \mathrm{~s}$ at $94^{\circ} \mathrm{C}$, $25 \mathrm{~s}$ of annealing at $\mathrm{Tm}$ under different primers, $30 \mathrm{~s}$ of extension at $72^{\circ} \mathrm{C}$, and a final extension for $10 \mathrm{~min}$ at $72^{\circ} \mathrm{C}$. All purified PCR products were bidirectionally sequenced by Sangon in a standard sequencing protocol. To test the availability of novel markers, we calculated the number of alleles, Fst, $\mathrm{Ho}$, and $\mathrm{He}$ of all microsatellite loci using GenAlEx version 6.3 (Peakall and Smouse, 2006) and the PIC values by PIC_CALC version 0.6 (Botstein et al., 1980). Hardy-Weinberg equilibrium (HWE) for each locus was calculated by Genepop version 4.1.4 (Yeh et al., 1997). The unweighted pair group mean analysis was estimated by TFPGA version 1.3 (Miller, 1997) with 5,000 permutations. Principal coordinates analysis (PCA) was visualized R software by ape package (Paradis et al., 2004).

\section{Cross-species Amplification and Transferability Analysis}

Eighteen related species of the genus Rhododendron, including seven species from subgen. Hymenanthes ( $R$. davidii, $R$. annae, $R$. denudatum, $R$. williamsianum, $R$. griersonianum, $R$. protistum var. giganteum, and $R$. delavayi), three species from subgen. Rhododendron ( $R$. ciliicalyx, $R$. siderophyllum, and $R$. yunnanense), two species from subgen. Tsutsusi ( $R$. simsii and $R$. pulchrum), two species from subgen. Azaleastrum ( $R$. hancockii and $R$. latoucheae), three species from subgen. Pseudorhodorastrum ( $R$. fuyuanense, $R$. spinuliferum, and $R$. spiciferum), and one species belonging to the subgen. Pentanthera ( $R$. molle), were chosen to evaluate the transferability of these newly developed EST-SSR markers. The genomic DNA extraction and PCR amplification were performed as previously described.

\section{RESULTS}

\section{Illumina Sequencing and de Novo Assembly}

A total of 56,989,279 paired-end raw reads that contain the adapter-primer sequences, low-quality sequences, and empty reads were generated to construct the cDNA library in Illumina NGS runs for $R$. rex. After a rigorous quality check and data filtering, 55,612,495 high-quality clean reads were obtained with 98.22\% Q20 and 94.69\% Q30 bases. The clean reads had a total nucleotide number of $8,238,889,450 \mathrm{nt}$, and the GC percentages for the clean reads were $49.77 \%$ (Table 1). Additionally, the highquality reads were deposited into the U.S. National Center for Biotechnology Information (NCBI) sequence read archive (SRA) database (Raw Illumina reads: NCBI SRP108011).

The total number of unigenes with paired-end reads was 164,242 , and the total length of the unigenes was $86,512,813$ bp, with an average length of 526.74 bp and the N50 and N90 value of 752 and $238 \mathrm{bp}$, respectively. In the 164,242 unigenes, the length of 111,599 unigenes $(67.95 \%)$ ranged from 1 to 400 bp; the length of 34,206 unigenes (20.83\%) ranged from 401 to $1,000 \mathrm{bp}$; the length of 17,853 unigenes $(10.88 \%)$ ranged from 1,001 to $4,000 \mathrm{bp}$; and 584 unigenes $(0.36 \%)$ had a length of more than $4,000 \mathrm{bp}$. The length distribution of unigenes is shown in Figure 1.

\section{Functional Annotation of Unigenes}

All nucleotide sequences were obtained by splicing, and Blast algorithm $(E<1 \mathrm{E}-5)$ were used to compare with $\mathrm{Nr}$, String, Swissprot, and KEGG database to obtain similarity sequence and the corresponding annotation information. Of the 164, 242 unigenes, 115,089 unigenes were successfully annotated in the $\mathrm{Nr}$, Pfam, Swiss-Prot, KEGG, COG, and GO databases, respectively (Supplementary Table S2, S3). 97,839 (59.57\%) unigenes were aligned to the $\mathrm{Nr}$ database, and 27,565 unigenes (28.17\%) of the control sequences had significant homology with the entries in 
TABLE 1 | Summary of the analysis of de novo assembled EST-SSRs for R. rex.

\begin{tabular}{llc}
\hline Category & Items & Number \\
\hline Raw reads & Total raw read & 56989279 \\
\hline Clean reads & Total clean reads & 55612495 \\
& Total clean nucleotides (nt) & 8238889450 \\
& Q20 percentage & $98.22 \%$ \\
& Q30 percentage & $94.69 \%$ \\
& GC percentage & $4 \%$ \\
\hline Unigenes & Total sequence number & 164242 \\
& Total sequence base & 86512813 \\
& Largest & 61022 \\
& Smallest & 201 \\
& Average & 526.74 \\
& N50(bp) & 752 \\
& N90 (bp) & 238 \\
\hline \multirow{2}{*}{ EST-SSR } & Total number of examined sequences & 164242 \\
& Total size of examined sequences (bp) & 86512813 \\
& Total number of identified SSRs & 15314 \\
& Number of SSR-containing sequences & 12188 \\
& Number of sequences containing more than one SSR & 2483 \\
& Number of SSRs present in compound formation & 1045 \\
& &
\end{tabular}

the Nr database ( $<1 \mathrm{E}-5)$, and nearly 62,992 unigenes (64.38\%) of the sequences showed more than $80 \%$ similarity (Supplementary Figure 1). A total of $62,018(37.76 \%)$ unigenes were assigned to the GO annotations, and these terms could be grouped into biological process $(168,696,46.61 \%)$, cellular component $(113,005,31.22 \%)$, and molecular function $(80,246,22,17 \%)$ of the three main categories to annotations (Figure 2). 17,247 unique sequences were assigned into the 25 COG categories. Of these classifications, the translation, ribosomal structure, and biogenesis only $(2,782,16.13 \%)$ represented the largest group, followed by general function prediction only $(1,883$, $10.91 \%$ ) and posttranslational modification, protein turnover, chaperones (1,775, 10.29\%) (Figure 3). 63,183 (38.47\%) unigenes had significant matches in the KEGG pathway database and were assigned to five main categories, including 389 KEGG pathways. In the total number of genes involved the pathway, in the five main categories, metabolism was the biggest category $(49,899$; $55.36 \%)$, followed by genetic information processing (16,536; $18.35 \%)$, organismal systems $(10,192 ; 11.31 \%)$, cellular processes $(6,971 ; 7.73 \%)$, and environmental information processing $(6,539 ; 7.25 \%)$ (Figure 4).

\section{Frequency and Distribution of SSRs in the Unigenes}

A total of 15,314 potential EST-SSRs were identified from 12,188 unigenes, with 2,483 unigenes containing more than one

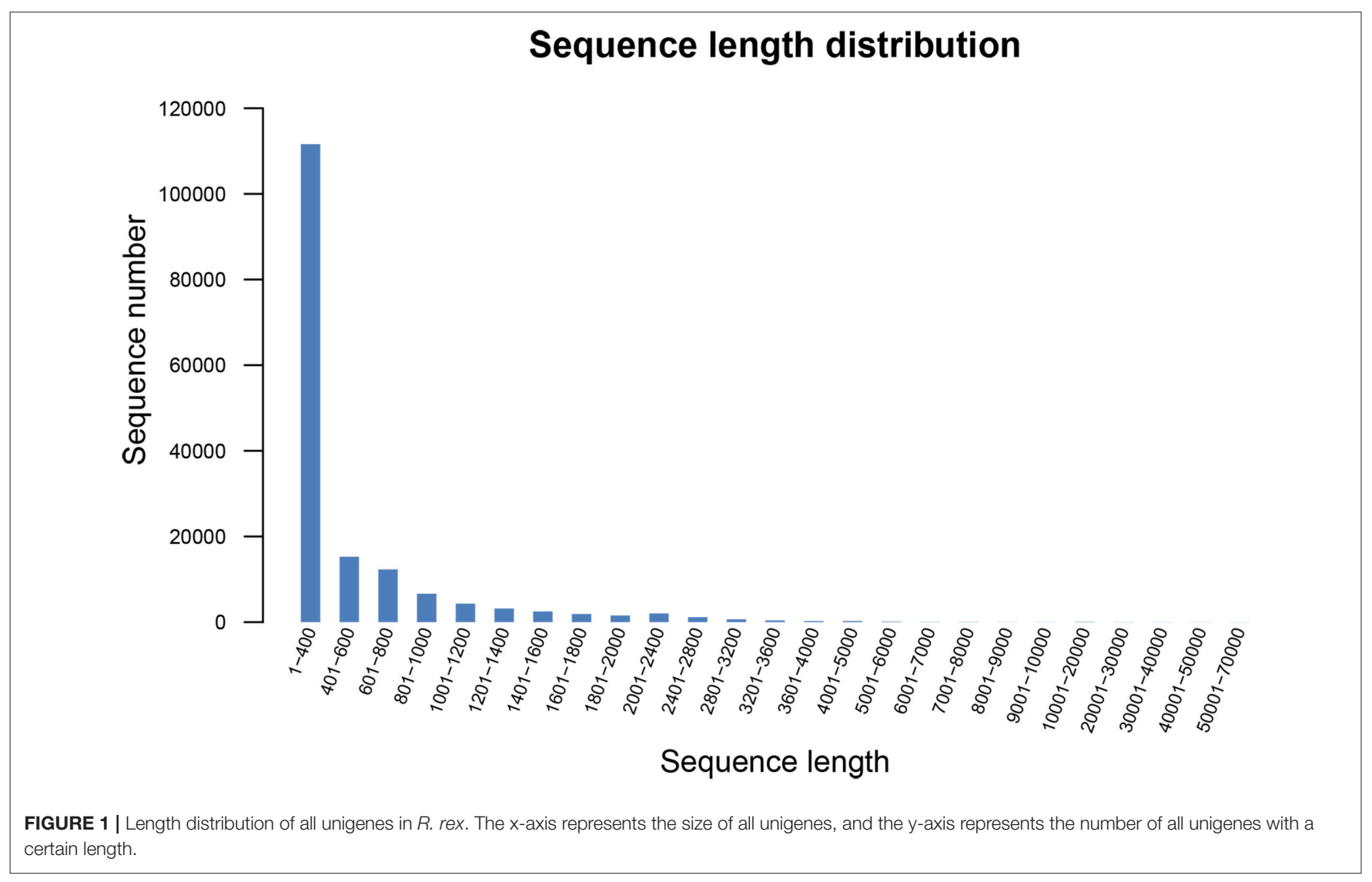




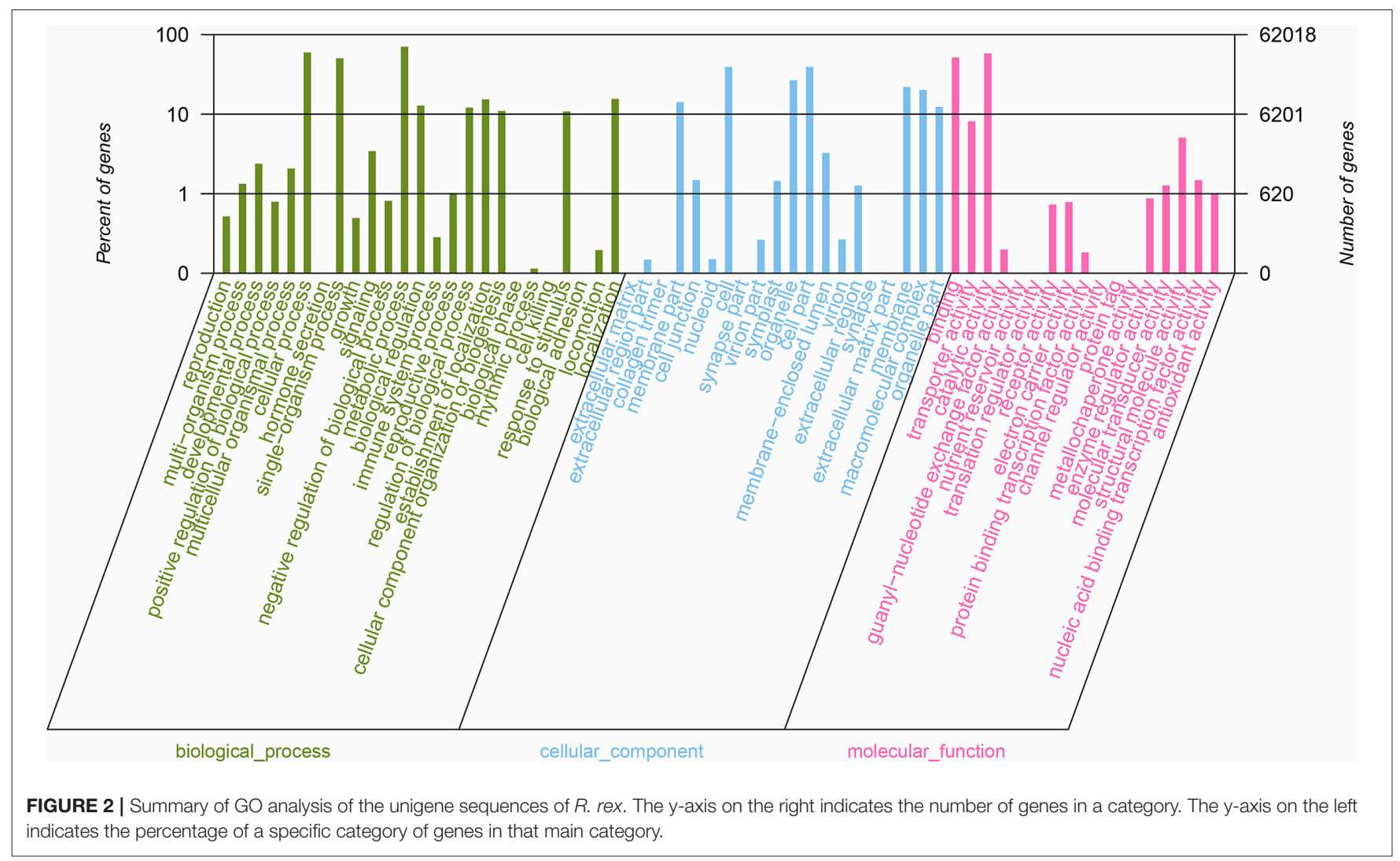

EST-SSR locus. Of the 15,314 EST-SSRs, 1,045 were compound microsatellites (Table 1). The frequency of SSRs in the R. rex unigenes was 9.32\%; an average of one EST-SSR was found every $5.65 \mathrm{~kb}$. The types and distributions of 15,314 potential ESTSSRs were investigated. The most abundant type was repeated dinucleotide $(8,366,54.63 \%)$, followed by mono- $(3,986,26.03 \%)$, tri- $(2,835,18.51 \%)$. Table 2 shows that the six tandem repeats of the EST-SSR $(2,533,16.54 \%)$ were the most common, followed by ten tandem repeats $(2,272,14.84 \%)$, nine tandem repeats $(2,088,13.63 \%)$, and eight, seven, and five tandem repeats were $(1,962,12.81 \%),(1,887,12.32 \%),(1,871,12.21 \%)$, whereas the rest of the tandem repeat each accounted for $<10 \%$ of the EST-SSR. The EST-SSR tandem repeat was 10-478 bp in length, and $18 \mathrm{bp}$ was the most frequently observed length (18.5\%).

In the di-nucleotide repeats, AG/CT was the most abundant motif $(7,632 ; 49.84 \%)$, followed by AC/GT (413; $2.7 \%), \mathrm{AT} / \mathrm{AT}(285 ; 1.86 \%)$, and CG/CG (36;0.24\%). In the tri-nucleotide repeats, the most dominant repeat motif was AAG/CTT (628; 4.1\%), followed by AGG/CCT (456; 2.98\%), AGC/CTG (425; 2.78\%), ACC/GGT (412; 2.69\%), CCG/CGG (256; 1.67\%), ATC/ATG (211; 1.38\%), AAC/GTT (211; 1.38\%), ACG/CGT (129; 0.84\%), AAT/ATT (59; 0.39\%), and ACT/AGT (48; 0.31\%) (Supplementary Table S4). The 14 repeat motifs mentioned above accounted for $73.16 \%$ of the total, whereas the remaining 56 types accounted for $26.84 \%$.

\section{Development and Validation of New EST-SSR Markers}

Based on the SSR-containing sequence, 100 primer pairs were randomly selected and synthesized and were successfully used for validation of the amplification and assessment of the polymorphism. In the selected 100 primer pairs, 50 were successfully amplified by $R$. rex genomic DNA, and the remaining 50 pairs of primers failed to amplify PCR products at various annealing temperatures. Among the 50 successful primer pairs, 45 produced the expected size of the amplified products, and the other five pairs of primers produced PCR products that were larger or smaller than expected. Using $20 R$. rex individuals from four populations as PCR templates, the results showed that 36 of the 45 primer pairs were polymorphic, and nine pairs were identified as monomorphic.

The 36 polymorphic EST-SSR markers developed in this study were used to assess the 20 genotype individuals. As presented in Table 3, 15 of 36 loci most significantly deviate from the equilibrium state in HWE test $(P<0.0001)$. A total of 197 alleles were detected. The number of alleles varied from 2 to 11 , with a mean of 5.472 alleles per locus. Observed heterozygosity ranged from 0.050 to 0.700 with a mean of 0.323 , and expected heterozygosity ranged from 0.045 to 0.698 with a mean of 0.372 . The PIC ranged from 0.154 to 0.870 , with a mean of 0.482 . The Fst ranged from 0.092 to 0.736 , with a mean of 0.268 . The null allele frequency ranged from 0.000 to 0.972 , with a mean of 0.263 (Table 3). In additional, the consequence of UPGMA clustering 


\section{COG Function Classification}
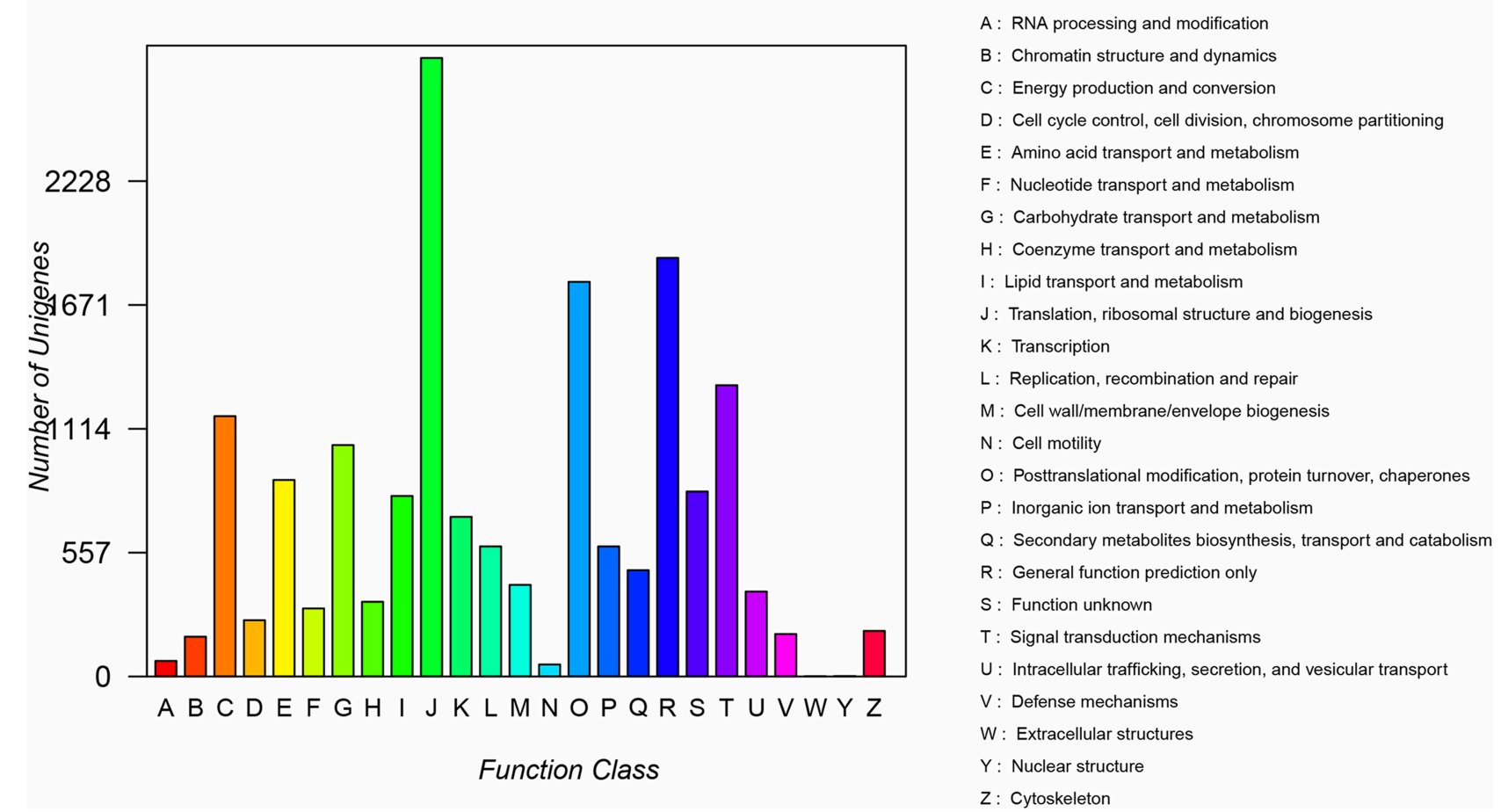

FIGURE 3 | COG analysis of the unigene sequences of $R$. rex. The $y$-axis indicates the number of unigenes in a specific functional cluster. The $x$-axis indicates the function class.

analysis and PCA analysis all showed that a distinctly genetic structure existed between the $4 R$. rex populations by the 36 novel EST-SSR markers (Supplementary Figures 2, 3).

\section{Transferability of the Newly Developed EST-SSR Markers}

A total of 36 pairs of primers were evaluated for transferability in 18 species (including seven species of subgen. Hymenanthes, three species of subgen. Rhododendron, two species of subgen. Azaleastrum, two species of subgen. Tsutsusi, three species of subgen. Pseudorhodorastrum, and one species of subgen. Pentanthera) of the genus Rhododendron. Nine primer pairs successfully amplified all of the templates. The transferability ratios ranged from 58.33 to $83.33 \%$ (subgen. Hymenanthes: 63.89\%; subgen. Rhododendron: 66.67\%; subgen. Azaleastrum: 83.33\%; subgen. Tsutsusi: 63.89\%; subgen. Pseudorhodorastrum: 58.33\%; and subgen. Pentanthera: $72.22 \%$ ).

\section{DISCUSSION}

\section{Characterization of the $R$. rex Transcriptome}

NGS has been increasingly used to analyze transcriptome sequencing and assembly in many plants because of its high efficiency, speed, accuracy, and low cost (Chen H. et al., 2015; An et al., 2016; Xing et al., 2017). SSR markers, due to their high polymorphism, high repeatability, and codominant inheritance, have been widely used to construct DNA fingerprints, genetic diversity analysis, gene mapping, and molecular marker-assisted breeding (Morgante et al., 1994; Agarwal et al., 2008; Luo et al., 2010). Deep transcriptome sequencing, which generates massive data, provides comprehensive information and a good resource for development of SSRs and gene discovery in many organisms (Zheng et al., 2013; Huang et al., 2014; Chen H. et al., 2015). Thus, markers based on transcriptome sequences have been successfully used for the detection of functional variation and gene-associated genetic analysis both in model and nonmodel species (Trick et al., 2009; Zheng et al., 2013; Yan et al., 2015). However, reports on transcriptome sequencing are few and available EST sequences of Rhododendron are also limited. In the present study, we first reported the transcriptome of R. rex by utilizing transcriptome sequences generated via NGS technologies. A total of 56,989,279 paired-end raw reads were generated and 55,612,495 high-quality clean reads were obtained with 98.22\% Q20 level, which ensures the quality of sequencing and is consistent with the results reported in Neolitsea sericea (Chen L. Y. et al., 2015) and Elymus sibiricus L. (Zhou et al., 2016). Compared with Quercus austrocochinchinensis (An et al., 2016) and $R$. latoucheae. (Xing et al., 2017), the mean N50 sizes (752 $\mathrm{bp)}$ of unigenes generated in this research were shorter. However, 164,242 unigenes were assembled from the $R$. rex transcriptome with an average length of $526.74 \mathrm{bp}$, which was longer than that 


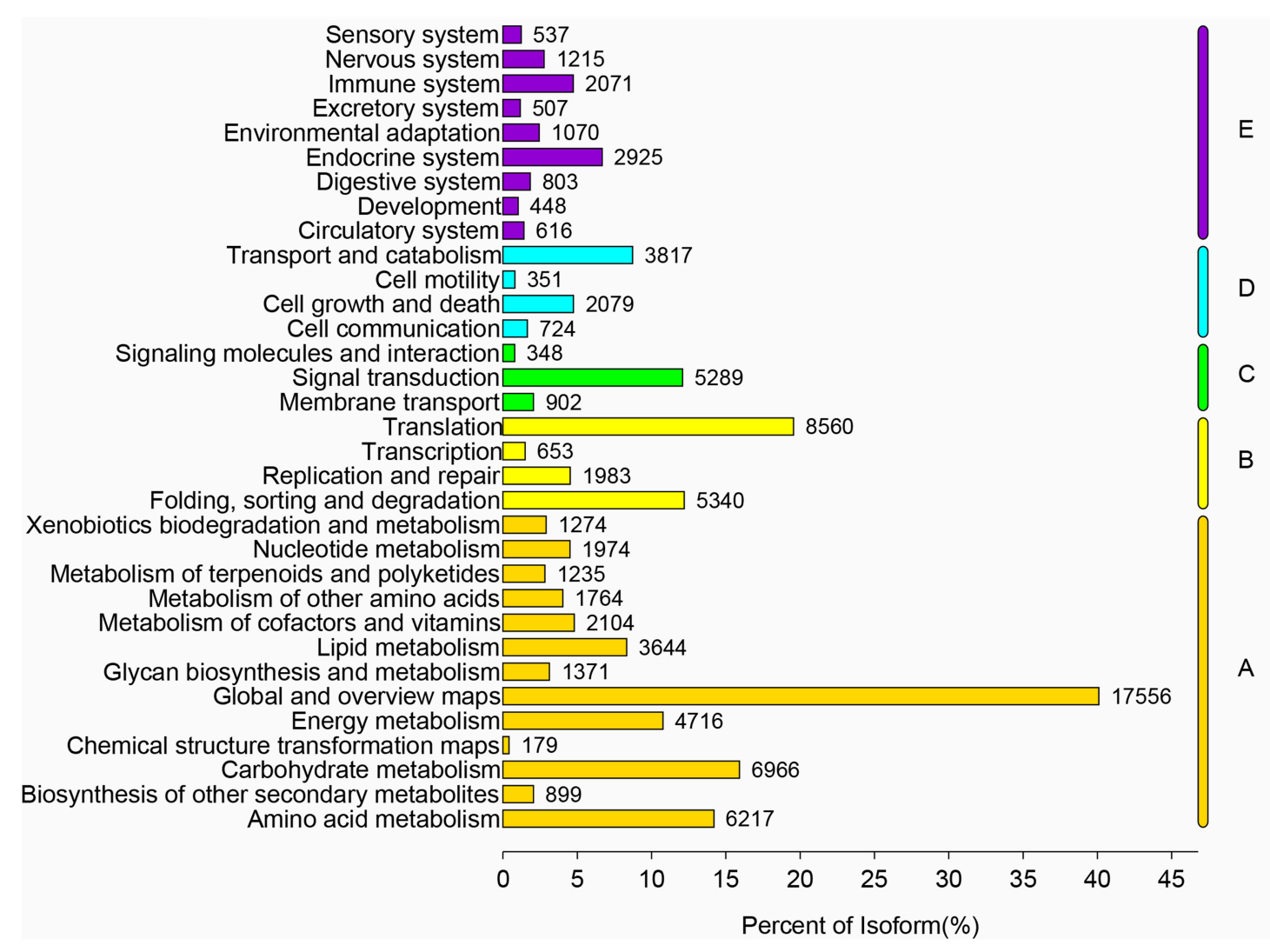

FIGURE 4 | KEGG metabolic pathway of $R$. rex. The $y$-axis is the name of the KEGG metabolic pathway, and the x-axis is the ratio of the number of genes and the number of genes to the number of genes.

reported in previous transcriptome studies of tea (402 bp; Tan et al., 2013), Hevea brasiliensis (485 bp; Li et al., 2012), and Rosa roxburghii (343 bp; Yan et al., 2015) but shorter than $N$. sericea (733 bp; Chen L. Y. et al., 2015), Cocos nucifera (752 bp; Xia et al., 2014), and Juglans mandshurica (752 bp; Hu et al., 2016). This result may be related to the difference between the assembler and the parameters and the differences in the nature of species. Overall, the large number of transcriptome sequences generated in the present study will be valuable for exploring the molecular mechanisms and for gene function.

The assembled unigenes of $R$. rex were successfully annotated to the known public databases Nr, Pfam, Swiss-Prot, KEGG, COG, and GO. These relatively complete annotations can provide valuable information for the future genetic diversity and evolutionary adaptation analysis of $R$. rex. In addition, some genes cannot match any functional annotations, possibly due to short sequence length discrepancies or limited annotation of the genus Rhododendron and related species in the current database (Xing et al., 2017). However, these failed annotations were still important resources for $R$. rex conservation or utilization. A large number of genomes are assigned to a wide range of GO categories, and COG classifications indicated that assembled unigenes represent a wide variety of transcripts in the $R$. rex genome. Of the three $\mathrm{GO}$ categories, metabolic process and cell under the biological process and cellular component were the largest group in our study, which were consistent with the report from $\mathrm{Li}$ et al. (2012). Meanwhile, we also found that some sequences in the GO database were annotated for the functions of response to temperature stimulus (GO: 0009266), photosynthesis (GO: 0015979), and osmotic stress (GO: 0006970), such as catalase (K03781) has been identified involved in plant cell growth as well as cold tolerance in other plants (Mutlu et al., 2013; Xu et al., 2013). Wei et al. (2005) also proposed that osmotic regulation, photoinhibition tolerance, and photosynthesis adjustment are important components for cold adaptation in Rhododendron. Thus, these sequences may represent a category of genes for cold adaptation and could be used to reveal evolutionary adaptation of cold tolerance mechanisms in a future study. In the COG classification, the first and second classifications found in our work were the translation, ribosomal structure, and biogenesis only and general function prediction only, which was similar to Yan et al. (2015) but was different from the report by Li et al. (2012). Moreover, 63,183 (38.47\%) unigenes had significant matches in the database and were assigned to five main categories, including 389 KEGG pathways. These results not only revealed the active metabolic processes in $R$. rex but also mean that various metabolites are synthesized in this species. 
TABLE 2 | Length distribution of the EST-SSRs of R. rex based on the number of nucleotide repeat units.

\begin{tabular}{|c|c|c|c|c|c|c|c|c|}
\hline Number of repeats & Mono- & Di- & Tri- & Quad- & Penta- & Hexa- & Total & Percentage (\%) \\
\hline 5 & & & 1,777 & 69 & 18 & 7 & 1,871 & 12.21 \\
\hline 6 & & 1,794 & 715 & 18 & & 6 & 2,533 & 16.54 \\
\hline 7 & & 1,566 & 317 & 2 & 2 & & 1,887 & 12.32 \\
\hline 8 & & 1,940 & 21 & & & 1 & 1,962 & 12.81 \\
\hline 9 & & 2,086 & 1 & & & 1 & 2,088 & 13.63 \\
\hline 10 & 1,418 & 853 & & & & 1 & 2,272 & 14.84 \\
\hline 11 & 787 & 121 & 1 & & & & 909 & 5.94 \\
\hline 12 & 509 & 6 & & & & & 515 & 3.36 \\
\hline 13 & 351 & & & & & & 351 & 2.29 \\
\hline 14 & 259 & & & & & & 259 & 1.69 \\
\hline 15 & 197 & & & & & & 197 & 1.29 \\
\hline 16 & 120 & & & & & & 120 & 0.78 \\
\hline 17 & 108 & & & & & & 108 & 0.71 \\
\hline 18 & 75 & & & & & 2 & 77 & 0.5 \\
\hline 19 & 47 & & 1 & & & & 48 & 0.31 \\
\hline 20 & 42 & & 1 & & & & 43 & 0.28 \\
\hline 21 & 38 & & & & & & 38 & 0.25 \\
\hline 22 & 26 & & & & & & 26 & 0.17 \\
\hline 23 & 8 & & & & & & 8 & 0.05 \\
\hline 24 & 1 & & 1 & & & & 2 & 0.01 \\
\hline Total & 3,986 & 8,366 & 2,835 & 89 & 20 & 18 & 15,314 & \\
\hline Percentage (\%) & 26.03 & 54.63 & 18.51 & 0.58 & 0.13 & 0.12 & & \\
\hline
\end{tabular}

\section{Frequency and Distribution of EST-SSRs}

The EST-SSR markers are important in the research of genetic diversity and population structure assessment in the species conservation, development of genetic maps, genomics comparison, and marker-assisted selection breeding (Chen L. Y. et al., 2015; Xing et al., 2017). In this study, a total of 15,314 potential EST-SSRs were identified from 12,188 unigenes, significantly improving the availability of SSR resources for marker development in this endangered and endemic species. Dinucleotide $(54.63 \%)$ repeats were the most abundant type, which was consistent with previous studies (Triwitayakorn et al., 2011; Yan et al., 2015; Xing et al., 2017), followed by mono- (26.03\%) and tri-nucleotide (18.51\%) repeats (Table 2). This result was consistent with previous findings that di-nucleotide repeats are the most common SSR repeat type in dicotyledonous plants (Kumpatla and Mukhopadhyay, 2005; Chen L. Y. et al., 2015). Studies have shown that the advantage of the di-nucleotide repeat sequence may be due to the over-expression of UTRs compared with open reading frames (Kumpatla and Mukhopadhyay, 2005; Qiu et al., 2010). In addition, the most abundant di-nucleotide repeats were AG/TC (49.84\%). The AG/CT motif can represent $\mathrm{UCU}$, and CUC codons in an mRNA population and translates into the amino acids Ala, and Leu which present in proteins at higher frequencies than other amino acids (Chen L. Y. et al., 2015). Thus, AG/CT motifs are present at high frequencies in EST collections of plants (Morgante et al., 2002; Chen L. Y. et al., 2015). CG/CG (0.24\%) was the least abundant motif in our result. It might be due to methylation of cytosine, which would inhibit the transcription of some plants (Chen L. Y. et al., 2015; Xing et al., 2017). In the tri-nucleotide repeats, the most dominant repeat motif was AAG/CTT (4.1\%). Previous studies of Arabidopsis, soybean, Cucumis sativus, Ricinus communis, Sesamum indicum, N. sericea also showed that the tri-nucleotide AAG motif may be significantly prominent in dicotyledonous plants (Morgante et al., 2002; Cavagnaro et al., 2010; Qiu et al., 2010; Wei et al., 2011; Chen L. Y. et al., 2015). The frequency of SSRs in the $R$. rex unigenes $(1 / 5.65 \mathrm{~kb})$ is much higher than those obtained in Elymus sibiricus (1/6.59 kb) (Zhou et al., 2016) and tree peony (1/9.24 kb) (Wu et al., 2014) but lower than the frequency of Camellia sinensis (1/4.99 kb) (Wu et al., 2013), $R$. latoucheae (1/2.87 kb) (Xing et al., 2017), and N. sericea (1/3.8 kb) (Chen L. Y. et al., 2015). The frequency is speculated to depend to some extent on the species differences that are also attributed to the SSR search criteria, database size, and the database-mining tools of different studies (Varshney et al., 2005; Biswas et al., 2012).

\section{Validation and Transferability of EST-SSR Makers}

In the selected 100 primer pairs that were randomly selected for PCR validation, 50 were successfully amplified by $R$. rex genomic DNA, and the remaining 50 pairs of primers failed to amplify PCR products at various annealing temperatures. 36 (36\%) were polymorphic among the four $R$. rex populations tested, and the remaining 64 pairs of primers failed to amplify PCR products at various annealing temperatures or produced 
TABLE 3 | Characteristics of the 36 novel EST-SSR markers in R. rex.

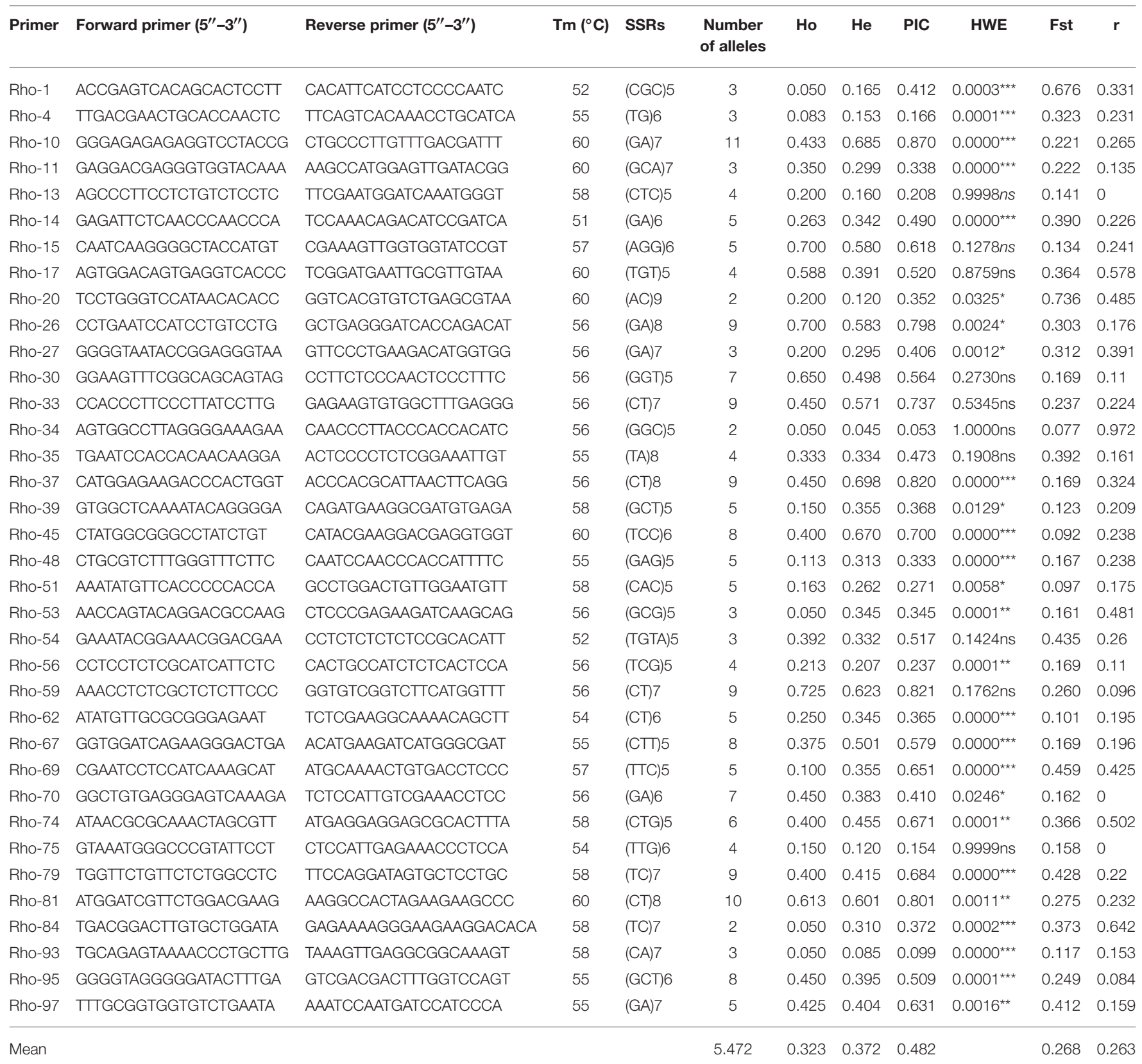

ns, non-significance; ${ }^{*} p<0.05$, significant difference; ${ }^{* *} p<0.01$, most significant difference; ${ }^{* * *} P<0.001$, most significant difference; $r$, null allele frequency.

PCR products that were larger or smaller than expected. This PCR success rate was higher than the rates reported for $N$. sericea (16.03\%) (Chen L. Y. et al., 2015), E. sibiricus (22.40\%) (Zhou et al., 2016), and J. mandshurica (30.8\%) (Hu et al., 2016), and Chrysanthemum nankingense (20\%) (Wang et al., 2013) but lower than the rates reported for tree peony (39.90\%) (Wu et al., 2014). Thus, the polymorphic ratio of EST-SSRs in this study was at a relatively high level. The level of polymorphism may be influenced by the amount of material used and the different geographic origin used in the study (Wu et al., 2014; Zhou et al., 2016).
Using the 36 polymorphic EST-SSR makers, 197 alleles were detected among the 20 R. rex individuals from four populations. The number of alleles per locus ranged from 2 to 11 with a mean of 5.47 alleles, expected heterozygosity $(\mathrm{He})$ averaging 0.372 , and observed heterozygosity $(\mathrm{Ho})$ averaging 0.323 , which is lower than that of the genomic SSRs in Lobelia deckenii (Ho $=0.762, \mathrm{He}=0.563)(\mathrm{Mi}$ et al., 2015) and Elymus sibiricus L. $(\mathrm{Ho}=0.49, \mathrm{He}=0.59)$ (Zhou et al., 2016). The PIC value of each EST-SSR reflects the allelic diversity and frequency of the sampled individuals and was used to evaluate the level of information (Botstein et al., 1980; Wu et al., 2014). In our 
study, PIC values ranged from 0.154 to 0.870 , averaging 0.482 , which present a moderate level. Thus, it indicated that the newly developed EST-SSR markers were an informative and effective tool for the genetic analysis, and evolutionary adaptation among a wide range of diverse Rhododendron species at the species level. However, since previous studies suggest that 20-30 individuals should be sampled in SSRs studies (Hale et al., 2012), we suggest that the species' genetic diversity and structure requires further investigation by expanding the sampling scale.

Previous studies have shown that the EST-SSR located in the transcribed region has a high degree of transferability (10-90\%) among the related species and established the range of variation of EST-SSR across species (Ellis and Burke, 2007; Wu et al., 2014). In the present study, the 36 polymorphic EST-SSR markers developed were used to evaluate for transferability in 18 species from six subgen. in the family Ericaceae. The transferability ratios ranged from 58.33 to $83.33 \%$, which is higher than that obtained in bottle gourd (4-41\%) (Xu et al., 2011), Elymus (49.11\%) (Zhou et al., 2016), and Cucumis (12.7\%) (Fernandez-Silva et al., 2008). Thus, the high transferability across the part of this genus in the present study will provide valuable sequence resources for the molecular marker development for Rhododendron species.

\section{CONCLUSION}

We used Illumina HiSeq4000 sequencing as well as de novo transcriptome assembly and annotation of $R$. rex. We also successfully identified and developed a set of EST-SSR markers in the present study. A total of 164, 242 unigenes with a mean length of $526.74 \mathrm{bp}$ from $R$. rex was generated. A total of 115,089 unigenes were successfully annotated into the $\mathrm{Nr}(97,839$ : 59.57\%), Pfam (37,981: 23.13\%), Swiss-Prot (60,600: 36.9\%), KEGG (63,183: 38.47\%), COG (17,247: 10.50\%), and GO (62,018: $37.76 \%)$ databases. Based on these unigene sequences, a total of 15,314 potential EST-SSRs were identified and characterized. A total of 100 primer pairs were randomly selected and synthesized

\section{REFERENCES}

Agarwal, M., Shrivastava, N., and Padh, H. (2008). Advances in molecular marker techniques and their applications in plant sciences. Plant Cell Rep. 27, 617-631. doi: 10.1007/s00299-008-0507-z

An, M., Deng, M., Zheng, S. S., and Song, Y. G. (2016). De novo transcriptome assembly and development of SSR markers of oaks Quercus austrocochinchinensis and Q. kerrii (Fagaceae). Tree Genet. Genomes 12:103. doi: 10.1007/s11295-016-1060-5

Biswas, M. K., Chai, L. J., Mayer, C., Xu, Q., Guo, W. W., and Deng, X. X. (2012). Exploiting BAC-end sequences for the mining, characterization and utility of new short sequences repeat (SSR) markers in Citrus. Mol. Biol. Rep. 39, 5373-5386. doi: 10.1007/s11033-0111338-5

Botstein, D., White, R. L., Skolnick, M., and Davis, R. W. (1980). Construction of a genetic linkage map in man using restriction fragment length polymorphisms. Am. J. Hum. Genet. 32, 314-331.

Camacho, C., Coulouris, G., Avagyan, V., Ma, N., Papadopoulos, J., Bealer, K., et al. (2009). BLAST+: architecture and applications. BMC Bioinform. 10:421. doi: 10.1186/1471-2105-10-421 and used for assessment of the polymorphism, thirty-six primer pairs were polymorphic and revealed polymorphism among 20 individuals from four $R$. rex populations. The newly developed EST-SSR markers have high transferability (58.33-83.33\%) in the present study. These results would not only provide an important resource for future genetic and genomic studies of $R$. rex but also represent a powerful molecular tool for evolutionary adaptation and genetic relationship analysis in other Rhododendron species.

\section{AUTHOR CONTRIBUTIONS}

SS, YZ, XZ, and YW initiated and designed the research. SS obtained funding for this study. YZ, XZ, and SS collected the materials and performed the experiments, SS, YZ, and XZ wrote and revised the paper. All authors read and approved the manuscript.

\section{FUNDING}

This study was financially supported by grant 31560224 and 31360155 from the National Natural Science Foundation of China and grant 2017YFC0505204 from the National Key Research and Development Project of China.

\section{ACKNOWLEDGMENTS}

We thank Fangli Liu, Xiongli Zhou, Guangsong Yang, and Yuanhuan Liu at Yunnan University for their help for in sample collection. We also thank Majorbio Bioinformatics (Shanghai, China) for technical support.

\section{SUPPLEMENTARY MATERIAL}

The Supplementary Material for this article can be found online at: http://journal.frontiersin.org/article/10.3389/fpls.2017. 01664/full\#supplementary-material
Cao, W. X., Zhang, D. G., and Hu, Z. Z. (2004). Ecological adaptability of four alpine species of the genus Rhododendron transplanted from higher to lower altitude. J. Gansu Agric. Univ. 1, 42-44. doi: 10.3969/jissn.1003-4315.2004.01.012

Cavagnaro, P. F., Senalik, D. A., Yang, L., Simon, P. W., Harkins, T. T., Kodria, C. D., et al. (2010). Genome-wide characterization of simple sequence repeats in cucumber (Cucumis sativus L.). BMC Genomics 11:569 doi: 10.1186/1471-2164-11-569

Chamberlain, D., Hyam, R., Argent, G., Fairweather, G., and Walter, K. S. (1996). "The genus Rhododendron: Its Classification and Synonymy. Edinburgh: The Royal Botanic Gardon Edinburgh Press.

Chen, H., Wang, L., Wang, S., Liu, C., Blair, M. W., and Cheng, X. (2015). Transcriptome sequencing of mung bean (Vigna radiate L.) genes and the identification of EST-SSR markers. PLOS ONE 10:e0120273. doi: 10.1371/journal.pone.0120273

Chen, L. Y., Cao, Y. N., Yuan, N., Nakamura, K., Wang, G. M., and Qiu, Y. X. (2015). Characterization of transcription and development of novel ESTSSR makers based on next-generation sequencing technology in Neolitsea sericea (Lauraceae) endemic to Asian land-bridge islands. Mol. Breed. 35:187. doi: 10.1007/s11032-015-0379-1 
Conesa, A., Götz, S., García-Gómez, J. M., Terol, J., Talón, M., and Robles, M. (2005). Blast2GO: a universal tool for annotation, visualization and analysis in functional genomics research. Bioinformatics 21, 3674-3676. doi: 10.1093/bioinformatics/bti610

Cordeiro, G. M., Casu, R., McIntyre, C. L., Manners, J. M., and Henry, R. J. (2001). Microsatellite markers from sugarcane (Saccharum spp.) ESTs cross transferable to erianthus and sorghum. Plant Sci. 160, 1115-1123. doi: 10.1016/S0168-9452(01)00365-X

Davey, J. W., Hohenlohe, P. A., Etter, P. D., Boone, J. Q., Catchen, J. M., and Blaxter, M. L. (2011). Genome-wide genetic marker discovery and genotyping using next-generation sequencing. Nat. Rev. Genet. 12:499. doi: 10.1038/nrg3012

Doyle, J. (1991). "DNA protocols for plants-CTAB total DNA isolation," in Molecular Techniques in Taxonomy, eds G. M. Hewitt and A. Johnston (Berlin: Springer), 283-293.

Ellis, J. R., and Burke, J. M. (2007). EST-SSRs as a resource for population genetic analyses. Heredity 99, 125-132. doi: 10.1038/sj.hdy.6801001

Fernandez-Silva, I., Eduardo, I., Blanca, J., Esteras, C., Pico, B., Nuez, F., et al. (2008). Bin mapping of genomic and EST-derived SSRs in melon (Cucumis melo L.). Theor. Appl. Genet. 118, 139-150. doi: 10.1007/s00122-0080883-3

Fu, L. G., and Jin, J. M. (1992). Red List of Endangered Plants in China. Beijing: Science Press.

Grabherr, M. G., Haas, B. J., Yassour, M., Levin, J. Z., Thompson, D. A., Amit, I., et al. (2011). Full-length transcriptome assembly from RNA-Seq data without a reference genome. Nat. Biotechnol. 29, 644-652. doi: 10.1038/nbt.1883

Hale, M. L., Burg, T. M., and Steeves, T. E. (2012). Sampling for microsatellitebased population genetic studies: 25 to 30 individuals per population is enough to accurately estimate allele frequencies. PLoS ONE 7:e45170. doi: 10.1371/journal.pone.0045170

Hart, R. (2015). Climate-Driven Change in Himalayan Rhododendron Phenology. St. Louis, MO: University of Missouri-Saint Louis.

Hu, Z., Zhang, T., Gao, X. X., Wang, Y., Zhang, Q., Zhou, H. J., et al. (2016). De novo assembly and characterization of the leaf, bud, and fruit transcriptome from the vulnerable tree Juglans mandshurica for the development of 20 new microsatellite markers using Illumina sequencing. Mol. Genet. Genomics 291, 849-862. doi: 10.1007/s00438-015-1147-y

Huang, D., Zhang, Y., Jin, M., Li, H., Song, Z., Wang, Y., et al. (2014). Characterization and high cross-species transferability of microsatellite markers from the floral transcriptome of Aspidistra saxicola (Asparagaceae). Mol. Ecol. Resour. 14, 569-577. doi: 10.1111/1755-0998.12197

Kumari, K., Muthamilarasan, M., Misra, G., Gupta, S., Subramanian, A., Parida, S. K., et al. (2013). Development of eSSR-markers in Setaria italica and their applicability in studying genetic diversity, cross-transferability and comparative mapping in millet and non-millet species. PLoS ONE 8:e67742. doi: 10.1371/journal.pone.0067742

Kumpatla, S. P., and Mukhopadhyay, S. (2005). Mining and survey of simple sequence repeats in expressed sequence tags of dicotyledonous species. Genome 48, 985-998. doi: 10.1139/g05-060

Li, D., Deng, Z., Qin, B., Liu, X. H., and Men, Z. H. (2012). De novo assembly and characterization of bark transcriptome using illumina sequencing and development of EST-SSR markers in rubber tree (Hevea brasiliensis Muell. Arg.). BMC Genomics 13:192. doi: 10.1186/1471-2164-13-192

Luo, R., Wu, W. L., Zhang, Y., and Li, Y. H. (2010). SSR marker and its application to crop genetics and breeding. Genomics Appl. Biol. 29, 137-143. doi: $10.3969 /$ gab.029.000137

Ma, Y. P., Wu, Z. K., Xue, R. J., Tian, X. L., Gao, L. M., and Sun, W. B. (2013). A new species of Rhododendron (Ericaceae) from the Gaoligong Mountains, Yunnan, China, supported by morphological and DNA barcoding data. Phytotaxa 114, 42-52. doi: 10.11646/phytotaxa.114.1.4

Mardis, E. R. (2008). Next-generation DNA sequencing methods. Ann. Rev. Genomics Hum. Genet. 9, 387-402. doi: 10.1146/annurev.genom.9. 081307.164359

Marsden, C. D., Woodroffe, R., Mills, M. G., McNutt, J. W., Creel, S., Groom, R., et al. (2012). Spatial and temporal patterns of neutral and adaptive genetic variation in the endangered African wild dog (Lycaon pictus). Mol. Ecol. 21, 1379-1393. doi: 10.1111/j.1365-294X.2012.05477.x

Mi, Q., Long, Z. C., Chen, J. M., Kamau, M. J., Chen, J. M., and Wang, Q. F. (2015). Development of SSR markers in Giant Lobelia (Lobelia deckenii) based on next-generation high-throughput sequencing. Plant Sci. J. 33, 847-854. doi: 10.11913/PSJ.2095-0837.2015.60847

Miller, M. P. (1997). Tools for population genetic analyses (TFPGA) 1.3: a Windows program for the analysis of allozyme and molecular population genetic data. Comput. Softw. 4:157.

Morgante, M., Hanafey, M., and Powell, W. (2002). Microsatellites are preferentially associated with nonrepetitive DNA in plant genomes. Nat. Genet. 30, 194-200. doi: 10.1038/ng822

Morgante, M., Rafalski, A., Biddle, P., Tingey, S., and Olivieri, A. M. (1994). Genetic mapping and variability of seven soybean simple sequence repeat loci. Genome 37, 763-769. doi: 10.1139/g94-109

Mutlu, S., Karadağoğlu, Ö., Atici, Ö., and Nalbantoğlu, B. (2013). Protective role of salicylic acid applied before cold stress on antioxidative system and protein patterns in barley apoplast. Biol. Plant 57, 507-513. doi: 10.1007/s10535-013-0322-4

Paradis, E., Claude, J., and Strimmer, K. (2004). APE: analyses of phylogenetics and evolution in R language. Bioinformatics 20, 289-290. doi: 10.1093/bioinformatics/btg412

Paux, E., Sourdille, P., Mackay, I., and Feuillet, C. (2012). Sequencebased marker development in wheat: advances and applications to breeding. Biotechnol. Adv. 30, 1071-1088. doi: 10.1016/j.biotechadv.2011. 09.015

Peakall, R., and Smouse, P. E. (2006). GENALEX 6: genetic analysis in excel. Population genetic software for teaching and research. Mol. Ecol. Notes 6, 288-295. doi: 10.1111/j.1471-8286.2005.01155.x

Qiu, L. J., Yang, C., Tian, B., Yang, J. B., and Liu, A. Z. (2010). Exploiting EST databases for the development and characterization of EST-SSR markers in castor bean (Ricinus communis L.). BMC Plant Biol. 10:278. doi: 10.1186/1471-2229-10-278

Shen, S. K., Wu, F. Q., Yang, G. S., Wang, Y. H., and Sun, W. B. (2015). Seed germination and seedling emergence in the extremely endangered species Rhododendron protistum var. giganteum-the world's largest Rhododendron. Flora 216, 65-70. doi: 10.1016/j.flora.2015.08.006

Song, Y. P., Jiang, X. B., Zhang, M., Wang, Z. L., Bo, W. H., An, X. M., et al. (2012). Differences of EST-SSR and genomic-SSR markers in assessing genetic diversity in poplar. For. Stud. China 14, 1-7. doi: 10.1007/s11632-0120106-5

Tan, L. Q., Wang, L. Y., Wei, K., Zhang, C. C., Wu, L. Y., Qi, G. N., et al. (2013). Floral transcriptome sequencing for SSR marker development and linkage map construction in the tea plant (Camellia sinensis). PLoS ONE 8:e81611. doi: 10.1371/journal.pone.0081611

Trick, M., Long, Y., Meng, J., and Bancroft, I. (2009). Single nucleotide polymorphism (SNP) discovery in the polyploid Brassica napus using Solexa transcriptome sequencing. Plant Biotechnol. J. 7, 334-346. doi: 10.1111/j.1467-7652.2008.00396.x

Triwitayakorn, K., Chatkulkawin, P., Kanjanawattanawong, S., Sraphet, S., Yoocha, T., Sangsrakru, D., et al. (2011). Transcriptome sequencing of Hevea brasiliensis for development of microsatellite markers and construction of a genetic linkage map. DNA Res. 18, 471-482. doi: 10.1093/dnares/dsr034

Varshney, R. K., Graner, A., and Sorrells, M. E. (2005). Genic microsatellite markers in plants: features and applications. Trends Biotechnol. 23, 48-55. doi: $10.1016 /$ j.tibtech.2004.11.005

Wang, H. B., Jiang, J. F., Chen, S. M., Qi, X. Y., Peng, H., Li, P. R., et al. (2013). Next-generation sequencing of the Chrysanthemum nankingense (Asteraceae) transcriptome permits large-scale unigene assembly and SSR marker discovery. PLoS ONE 8:e62293. doi: 10.1371/journal.pone.0062293

Wei, H., Dhanaraj, A. L., Rowland, L. J., Fu, Y., Krebs, S. L., and Arora, R. (2005). Comparative analysis of expressed sequence tags from cold-acclimated and non-acclimated leaves of Rhododendron catawbiense Michx. Planta 221, 406-416. doi: 10.1007/s00425-004-1440-1

Wei, W., Qi, X., Wang, L., Zhang, Y., Hua, W., Li, D., et al. (2011). Characterization of the sesame (Sesamum indicum L.) global transcriptome using Illumina paired-end sequencing and development of EST-SSR markers. BMC Genomics 12:451. doi: 10.1186/1471-2164-12-451

Wu, F. Q., Shen, S. K., Zhang, X. J., Wang, Y. H., and Sun, W. B. (2015). Genetic diversity and population structure of an extremely endangered species: the world's largest Rhododendron. AoB Plants 7:plu082. doi: 10.1093/aobpla/plu082 
Wu, H. L., Chen, D., Li, J. X., Yu, B., Qiao, X. Y., Huang, H. L., et al. (2013). De novo characterization of leaf transcriptome using 454 sequencing and development of EST-SSR markers in tea (Camellia sinensis). Plant Mol. Biol. Rep. 34, 524-538. doi: 10.1007/s11105-012-0519-2

Wu, J., Cai, C. F., Cheng, F. Y., Cui, H. L., and Zhou, H. (2014). Characterisation and development of EST-SSR markers in tree peony using transcriptome sequences. Mol. Breed. 34, 1853-1866. doi: 10.1007/s11032-0140144-x

Xia, W., Xiao, Y., Liu, Z., Luo, Y., Mason, A. S., Fan, H. K., et al. (2014). Development of gene-based simple sequence repeat markers for association analysis in Cocos nucifera. Mol. Breed. 34, 525-535. doi: 10.1007/s11032-014-0055-X

Xing, W., Liao, J. Y., Cai, M. Y., Xia, Q. F., Liu, Y., and Zeng, W. (2017). De novo assembly of transcriptome from Rhododendron latoucheae Franch. using Illumina sequencing and development of new EST-SSR markers for genetic diversity analysis in Rhododendron. Tree Genet. Genomes 13:53. doi: 10.1007/s11295-017-1135-y

Xu, J., Duan, X., Yang, J., Beeching, J. R., and Zhang, P. (2013). Coupled expression of $\mathrm{Cu} / \mathrm{Zn}$-superoxide dismutase and catalase in cassava improves tolerance against cold and drought stresses. Plant Signal. Behav. 8:e24525. doi: $10.4161 /$ psb. 24525

Xu, P., Wu, X., Luo, J., Wang, B., Liu, Y., Ehlers, J. D., et al. (2011). Partial sequencing of the bottle gourd genome reveals markers useful for phylogenetic analysis and breeding. BMC Genomics 12:467. doi: 10.1186/1471-2164$12-467$

Yan, X., Zhang, X., Lu, M., He, Y., and An, H. (2015). De novo sequencing analysis of the Rosa roxburghii fruit transcriptome reveals putative ascorbate biosynthetic genes and EST-SSR markers. Gene 561, 54-62. doi: 10.1016/j.gene.2015.02.054
Ye, J., Fang, L., Zheng, H., Zhang, Y., Chen, J., Zhang, Z., et al. (2006). WEGO: a web tool for plotting GO annotations. Nucleic Acids Res. 34, W293-W297. doi: 10.1093/nar/gkl031

Yeh, F. C., Yang, R., Boyle, T. B., Ye, Z., and Mao, J. X. (1997). PopGene, the UserFriendly Shareware for Population Genetic Analysis. Alberta: Molecular Biology and Biotechnology Center, Centre University of Alberta.

Yoichi, W., Sakaguchi, S., Ueno, S., Tomaru, N., and Uehara, K. (2016). Development and characterization of EST-SSR markers for the genus Rhododendron section Brachycalyx (Ericaceae). Plant Spec. Biol. doi: 10.1111/1442-1984.12155

Zheng, X., Pan, C., Diao, Y., You, Y., Yang, C., and Hu, Z. (2013). Development of microsatellite markers by transcriptome sequencing in two species of Amorphophallus (Araceae). BMC Genomics 14:490. doi: 10.1186/1471-2164-14-490

Zhou, Q., Luo, D., Ma, L. C., Xie, W. G., Wang, Y., Wang, Y. R., et al. (2016). Development and cross-species transferability of EST-SSR markers in Siberian wildrye (Elymus sibiricus L.) using Illumina sequencing. Sci. Rep. 6:20549. doi: $10.1038 /$ srep20549

Conflict of Interest Statement: The authors declare that the research was conducted in the absence of any commercial or financial relationships that could be construed as a potential conflict of interest.

Copyright (c) 2017 Zhang, Zhang, Wang and Shen. This is an open-access article distributed under the terms of the Creative Commons Attribution License (CC BY). The use, distribution or reproduction in other forums is permitted, provided the original author(s) or licensor are credited and that the original publication in this journal is cited, in accordance with accepted academic practice. No use, distribution or reproduction is permitted which does not comply with these terms. 